\title{
Publisher's Note: Intrinsic antiferromagnetic coupling underlies colossal magnetoresistance effect: Role of correlated polarons [Phys. Rev. B 89, 024420 (2014)]
}

\author{
V. Moshnyaga, A. Belenchuk, S. Hühn, C. Kalkert, M. Jungbauer, O. I. Lebedev, S. Merten, K.-Y. Choi, P. Lemmens, \\ B. Damaschke, and K. Samwer \\ (Received 30 December 2014; published 6 January 2015)
}

DOI: 10.1103/PhysRevB.91.019901

PACS number(s): 75.47.Lx, 71.38.-k, 75.47.Gk, 99.10.Fg

This paper was published online on 30 January 2014 with an omission in the Acknowledgments. On page 7, "S.M. and V.M. thank B. Schmidt from Geowissenschaftlihes Zentrum at the University of Göttingen for giving the opportunity and help with Raman measurements" has been added to the Acknowledgments. The Acknowledgments have been corrected as of 24 December 2014. The Acknowledgments are incorrect in the printed version of the journal. 